Association for Information Systems AIS Electronic Library (AISeL)

Wirtschaftsinformatik Proceedings 2005

Wirtschaftsinformatik

February 2005

\title{
Indikatoren zur Bewertung der Nachhaltigkeit von Unternehmensarchitekturen
}

\author{
Stephan Aier \\ Technische Universität Berlin \\ Turgut Dogan \\ Universität Potsdam
}

Follow this and additional works at: http://aisel.aisnet.org/wi2005

\section{Recommended Citation}

Aier, Stephan and Dogan, Turgut, "Indikatoren zur Bewertung der Nachhaltigkeit von Unternehmensarchitekturen" (2005).

Wirtschaftsinformatik Proceedings 2005. 32.

http://aisel.aisnet.org/wi2005/32

This material is brought to you by the Wirtschaftsinformatik at AIS Electronic Library (AISeL). It has been accepted for inclusion in Wirtschaftsinformatik Proceedings 2005 by an authorized administrator of AIS Electronic Library (AISeL). For more information, please contact elibrary@aisnet.org. 
In: Ferstl, Otto K, u.a. (Hg) 2005. Wirtschaftsinformatik 2005: eEconomy, eGovernment, eSociety; 7. Internationale Tagung Wirtschaftsinformatik 2005. Heidelberg: Physica-Verlag

ISBN: 3-7908-1574-8

(C) Physica-Verlag Heidelberg 2005 


\title{
Indikatoren zur Bewertung der Nachhaltigkeit von Unternehmensarchitekturen
}

\author{
Stephan Aier \\ Technische Universität Berlin \\ Turgut Dogan \\ Universität Potsdam
}

Zusammenfassung: Nachhaltigkeit ist ein aus der Umweltökonomie stammendes Konzept, welches angewandt auf Unternehmen einen verantwortungsvollen Umgang mit der physischen (Ökologie) und sozialen Umwelt (Ökonomie, Soziologie) fordert. Dieser Beitrag diskutiert, inwiefern sich Nachhaltigkeit, losgelöst von Fragen der Ökologie, auf Unternehmensarchitekturen übertragen lässt und welche Indikatoren sich für ein Bewertungssystem nachhaltiger Architekturen eignen.

Schlüsselworte: Nachhaltigkeit, Nachhaltigkeitsindikatoren, Unternehmensarchitektur, Systemintegration, Flexibilisierung, Modularisierung, EAI

\section{Problemstellung}

Wandel ist zu einem Dauerzustand in Unternehmen geworden [Krue98, S. 228ff]. Dies betrifft alle Bereiche von Unternehmen. Produktlebenszyklen - insbesondere technischer Produkte - sind erheblich kürzer geworden. Die Änderungszyklen der die Produktentwicklung und Produktion begleitenden Prozesse ebenso. Neue Technologien wie das Internet haben deutliche Auswirkungen auf die Prozessgestaltung der Leistungserstellung, die Integration mit Lieferanten und die Kommunikation mit Kunden [FrSt02]. Dazu kommen immer neue Managementmoden, Informationstechnologien und rechtliche Rahmenbedingungen [KiWa03, S. 410ff].

Dieser permanente Wandel führt zu häufigen Reorganisationen verbunden mit der Anpassung und Ablösung der die Prozesse unterstützenden IT-Systeme. Gab es in der Vergangenheit die Möglichkeit, die Unternehmung in entsprechenden Abständen grundlegend neu auf die veränderte Situation auszurichten, ist das bei der aktuellen Veränderungsrate weder durchführbar noch effizient [ $\left.\mathrm{Neu}^{+} 00\right]$.

In diesem Beitrag soll darum diskutiert werden, inwieweit das Konzept der Nachhaltigkeit ein geeignetes Gestaltungsziel für die Architektur der Unternehmung 
darstellt, um diese in die Lage zu versetzen, in effizienter Art und Weise mit permanentem Wandel umzugehen. Es sollen Ansätze zur Bewertung der Nachhaltigkeit von Unternehmensarchitekturen aufgezeigt werden.

Dazu wird im folgenden Abschnitt das diesem Beitrag zu Grunde liegende Verständnis von Unternehmensarchitektur erläutert. Danach werden verschiedene Definitionen der Nachhaltigkeit betrachtet, die ihren Ursprung in der Nachhaltigkeitsdebatte der Umweltökonomie haben. Es wird diskutiert, inwieweit sich Teile dieses Nachhaltigkeitsbegriffs auf die Wirtschaftsinformatik übertragen lassen. Darauf aufbauend werden Ansätze für die Gestaltung und die Bewertung nachhaltiger Architekturen vorgestellt.

\section{Verständnis der Unternehmensarchitektur}

Der Begriff der Unternehmensarchitektur ist zentraler Bestandteil dieses Beitrags. Verkürzt kann eine Architektur als eine abstrakte, ganzheitliche Betrachtung von Strukturen und Mustern mit Planungscharakter aufgefasst werden $\left[\mathrm{Bas}^{+} 03, \mathrm{~S}\right.$. 19ff]. Architekturen sind in der Regel das Ergebnis eines Planungsprozesses und stellen nach ihrer Definition selbst einen Masterplan für die ganzheitliche Realisierung zukünftiger Maßnahmen dar.

Diese allgemeinen Charakteristika lassen sich auf die Gestaltung von Unternehmen anwenden und werden so zur Unternehmensarchitektur. Unter einer Unternehmensarchitektur wird das Zusammenwirken organisatorischer, technischer und psychosozialer Aspekte bei der Planung und Entwicklung betrieblicher soziotechnischer Informationssysteme verstanden [Gron03, S. 45]. Im Folgenden sollen vor allem die organisatorische und die technische Dimension der Unternehmensarchitektur erläutert werden. Dazu werden die Begriffe Organisationsarchitektur und IT-Architektur verwendet (Abbildung 1).

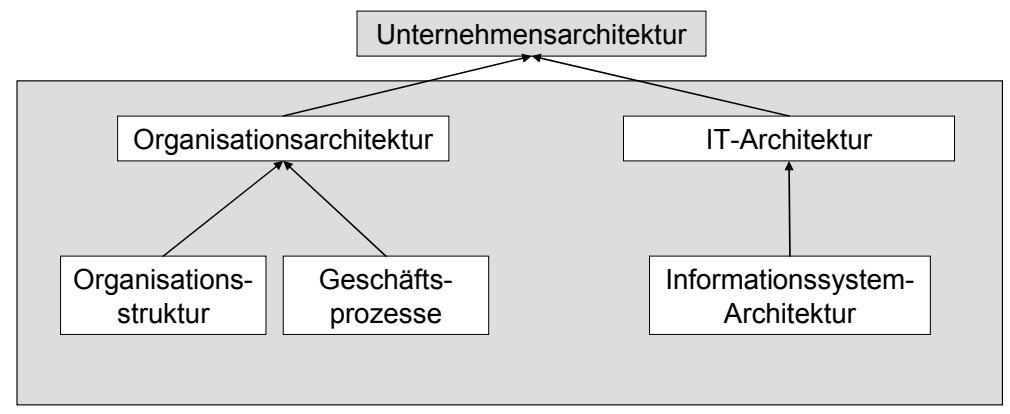

Abbildung 1: Bestandteile der Unternehmensarchitektur 
Die Organisationsarchitektur enthält alle nichttechnischen Bestandteile der Unternehmensarchitektur und kann mit dem instrumentalen Organisationsbegriff verglichen werden, der die Gesamtheit aller generellen expliziten Regelungen zur Gestaltung der Aufbau- und Ablauforganisation umfasst [Blei91, S. 35]. Entsprechend wird die Organisationsarchitektur hier in die Organisationsstruktur und die Geschäftsprozesse unterschieden.

Komplementär zur Organisationsarchitektur steht die IT-Architektur. Sie umfasst alle technischen Bestandteile der Unternehmensarchitektur. Insbesondere beinhaltet sie die technischen Informationssysteme, welche ihrerseits eine eigene Architektur, die Informationssystemarchitektur, aufweisen.

In der Literatur sind die Begriffe Organisationsarchitektur und IT-Architektur häufig verwendet, jedoch meist anders definiert. Abhängig von der fachlichen Herkunft des Autors umfasst die Organisationsarchitektur auch technische Komponenten [ $\left.\mathrm{Nad}^{+} 94\right]$ bzw. die IT-Architektur auch organisatorische Fragestellungen [Krcm90]. Im Folgenden sollen jedoch beide Bereiche separat und somit bezogen auf ihre Bedeutung gleichwertig betrachtet werden. Dies ist sinnvoll, da beide eine hohe Bedeutung für das effiziente Funktionieren einer Unternehmung haben und zwischen ihnen komplexen Wechselwirkungen bestehen [AiSc03, S. 19ff].

Dass bei der Gestaltung effizienter, nachhaltiger Unternehmen die Unternehmungsorganisation Gegenstand der Betrachtung sein muss, ist evident. Die Notwendigkeit der Betrachtung der Unternehmungs-IT resultiert aus dem Stellenwert der IT für die operative Prozessabwicklung. Der Dienstleistungsbereich, z.B. die Bankenbranche, weist diesbezüglich eine besondere Relevanz auf, da hier praktisch alle Geschäftsprozesse durch eine starke IT-Unterstützung gekennzeichnet sind. MORGAN spricht in diesem Zusammenhang von der annähernden Gleichbedeutung von IT und Unternehmung [Morg97, S. 117].

\section{Begriff der Nachhaltigkeit}

Im folgenden Kapitel sollen im ersten Teil die vielschichtigen Definitionen des Nachhaltigkeitsbegriffs systematisiert werden, um dann im zweiten Teil deren Anwendbarkeit und Ausprägung für den Bereich der Wirtschaftsinformatik darzulegen.

\subsection{Definitionen des Nachhaltigkeitsbegriffs}

Bei der Analyse der Definitionen und Ansätze der Nachhaltigkeit und nachhaltigen Entwicklung ist die Herkunft dieses Begriffs aus der Umweltökonomie nicht zu übersehen. Die wohl meist verbreitete Definition stammt von der WORLD 
COMMISSION ON ENVIRONMENT AND DEVELOPMENT aus ihrem Bericht „Our Common Future ", der als der so genannte Brundtland Report bekannt wurde:

"Sustainable development seeks to meet the needs and aspirations of the present without compromising the ability to meet those of the future." [Worl87, S. 43]

Es existiert eine beliebige Anzahl weiterer Definitionen von Nachhaltigkeit bzw. nachhaltiger Entwicklung. ,Als weitgehend konsensfähige, hochabstrakte, normative, anthropozentrische Leit- und Wertidee, die konkrete Handlungen gerade nicht festlegt, entzieht sich sustainable development ... zunächst einer präzisen eindeutigen Definition“ [Conr00, S. 2]. Die Anwendung des Konzepts setzt voraus, dass in Bezug auf den jeweiligen Kontext dargelegt wird, was unter nachhaltiger Entwicklung bzw. Nachhaltigkeit genau verstanden wird. Eine Auswertung der wissenschaftlichen Literatur, in der Definitionen des Begriffs vorgenommen wurden, zeigt eine außerordentliche Spannbreite hinsichtlich der Frage, auf welche Gegenstandsbereiche sich der Begriff beziehen soll - eine einheitliche Definition ist nicht vorhanden [Teic02, S. 16f].

HUBER bündelt basierend auf der Analyse der relevanten Literatur Nachhaltigkeit in die drei Strategien Effizienz, Suffizienz und Konsistenz [Hube95, S. 39], GRONAU fügt die Strategie der Partizipation hinzu [Gron03, S. 222].

Der Suffizienzstrategie liegt die Frage zu Grunde: Wieviel ist genug? Eine Antwort lässt sich nur schwer finden, auf jeden Fall scheint es aber angebracht, genügsam zu sein. Suffizienz erhebt die Forderung, durch Verzicht ein ausreichendes Maß an Genügsamkeit zu realisieren. Die Kritik an dieser Strategie lautet: Sie ist unrealistisch und mit Fehlwirkungen behaftet - ersteres, weil sie der allgemeinen Norm der individuellen Nutzenmaximierung zuwider läuft. Fehlwirkungen weist sie auf, weil Beschränkung zu wirtschaftlicher und gesellschaftlicher Stagnation oder zumindest zu Fehlentwicklungen in diesen Bereichen führen kann. Dem kann entgegen gehalten werden, dass es Unbeschränktheit nicht gibt und jedes System Grenzen in Raum und Zeit aufweist. Für eine erfolgreiche Umsetzung der Suffizienzstrategie ist es empfehlenswert, ökonomische statt moralische Anreize zu setzen [Hube95, S. 40].

Die Effizienzstrategie zielt auf die Steigerung der (Ressourcen-) Produktivität, um dadurch Leistungen wirtschaftlich, d.h. mit dem kleinsten möglichen Ressourcenverbrauch zu erstellen. Zentrale Konzepte sind dabei Wiederverwendung und Langlebigkeit. Die Effizienzstrategie ist die für das Wirtschaftsgeschehen anschlussfähigste Strategie, weshalb sie hier auch oft mit Nachhaltigkeit verwechselt wird. Effizienz nimmt eine Mittlerstellung zwischen Suffizienz und Konsistenz ein.

Die Konsistenzstrategie hat entweder die vollständige Abschirmung von Systemen von deren Umwelt oder die Sicherstellung deren Verträglichkeit, Stimmigkeit mit dem sie umgebenden (Super-)System zum Ziel [Hube95, S. 41f]. Wenn Systeme keine Verbindungen zur umgebenden Umwelt haben, müssen diese Systeme auch 
nicht mit der Umwelt verträglich sein, da es keine Wirkungsbeziehungen zwischen ihnen gibt. Bestehen jedoch Beziehungen, so wird Konsistenz der Systeme mit ihrer Umwelt gefordert.

Die Partizipationsstrategie schließlich fordert die Teilhabe, d.h. die Einbindung der von einer Systemgestaltung Betroffenen. Dies ist nötig, um das System zum einen bestmöglich für die „Betroffenen“ zu gestalten und zum anderen, um deren Akzeptanz des Systems sicherzustellen [Gron03, S. 222].

\subsection{Nachhaltigkeitsbegriff der Wirtschaftsinformatik}

Wird Nachhaltigkeit als ein für die Unternehmung relevantes Thema betrachtet, so geht es meist um die Reduktion negativer externer Effekte auf die Umwelt oder die Gesellschaft (physische und soziale Umwelt), welche durch das Handeln des Unternehmens entstehen [LeSt03, S. 259f]. Dies soll im Folgenden als extern orientierte Nachhaltigkeit bezeichnet werden.

Das hier zu Grunde gelegte Verständnis von Nachhaltigkeit soll vollkommen losgelöst von jedweden Themen der Ökologie sein. HAHN/HUNGENBERG definieren das Oberziel einer jeden Unternehmung als Erhaltung und erfolgreiche Weiterentwicklung, als Erfüllung der Individualziele aller an der Unternehmung interessierten Gruppen [HaHu01, S. 13]. Hier soll das Ziel der Betrachtung eine unternehmensinterne Sicht der Nachhaltigkeit mit dem primären Ziel der langfristigen, effizienten Unternehmensführung und -erhaltung sein. Diese effiziente Unternehmenserhaltung, d.h. Systemerhaltung mit minimalem Aufwand, kann als ein Unterziel dieses Oberziels betrachtet werden. Dies soll im Folgenden als intern orientierte Nachhaltigkeit bezeichnet werden.

Ziel dieses Beitrags ist es nicht, eine geschlossene Theorie nachhaltiger Architekturen im Rahmen der Wirtschaftsinformatik darzulegen. Es lassen sich jedoch gemeinsame Definitionsmerkmale mit dem extern orientierten Nachhaltigkeitsbegriff herausstellen. Der wichtigste Punkt ist der Erhalt des Systems - hier der Unternehmung mit ihren wesentlichen Bestandteilen. Unklar ist jedoch, wie lange ein System erhalten bleiben soll und wie Nachhaltigkeit überprüft werden kann. Meist wird von langfristiger Erhaltung des Systems gesprochen. Da jedes System jedoch endlich ist, gibt es auch keine unbegrenzte Nachhaltigkeit. Weiterhin ist die Beurteilung von Nachhaltigkeit nur nachträglich möglich, da zukünftige Entwicklungen oft nur schwer prognostizierbar sind. Als Konsequenz daraus können keine operativen Handlungsanweisungen gegeben werden, die mit Sicherheit zu Nachhaltigkeit führen [Krca03, S. 18f].

Dennoch werden im folgenden Abschnitt einige Strategien eines intern orientierten Nachhaltigkeitsbegriffs erläutert. In Abschnitt 5 wird das Problem der nachträglichen Beurteilung von Nachhaltigkeit diskutiert. 


\section{Nachhaltigkeitsstrategien}

Die im vorangegangenen Abschnitt dargestellten Ansätze zur Gewinnung intern orientierter Nachhaltigkeit bedürfen der operativen Konkretisierung durch Handlungsstrategien. Im Folgenden sollen einige Ansatzpunkte für die Gestaltung nachhaltiger Organisations- und IT-Architekturen, sowie integrierte Strategien zur Gestaltung nachhaltiger Unternehmensarchitekturen genannt werden (vgl. zu einer ausführlichen Darstellung [AiDo04].).

\section{1 Übertragung klassischer Nachhaltigkeitsstrategien}

Zunächst lassen sich HUBERs klassische Nachhaltigkeitsstrategien auf die Organisation und die IT übertragen. Aspekte der Konsistenzstrategie sind auf Seiten der Organisation im interdependenten Charakter der Teildimensionen der Organisationsarchitektur wieder zu finden. Es herrscht Einigkeit darüber, dass Organisationsstruktur und die darin ablaufenden Geschäftsprozesse nicht unabhängig voneinander gestaltet werden können, da es sich um unterschiedliche Sichtweisen auf den gleichen Betrachtungsgegenstand handelt. Daher würde eine mangelnde Stimmigkeit zwischen beiden Dimensionen zu Ineffizienzen führen [Blei91, S. 42]. Aufgegriffen wurden solche Überlegungen z.B. durch die verstärkte Prozessorientierung, durch welche vor allem die für die Abläufe hinderlichen Organisationsbrüche vermindert werden sollten [Gait83]. Ebenso ist auf Seiten der IT eine Konsistenz zwischen den Informationssystemen notwendig. Auf die schließlich notwendige Konsistenz zwischen Organisations- und IT-Architektur wird in Abschnitt 4.4 eingegangen.

FRESE untergliedert organisatorische Effizienz in Koordinations- und Motivationseffizienz. Koordinationseffizienz wird durch ein gemeinsames Minimum an Autonomie- und Abstimmungskosten erreicht [Fres00, S. 258ff]. Ein unverändert aktueller Ansatz, die organisatorische Effizienz zu optimieren, ist es, den Organisationsmitgliedern ein hohes Maß an Autonomie zu gewähren. Sowohl die Organisationsstruktur als auch die Geschäftsprozesse sollen die Selbststeuerung der Organisationselemente unterstützen und damit den Grad der Formalisierung senken [KiKu92, S. 467ff]. Auf Seiten der IT fordert die Effizienzstrategie einen minimalen Anpassungsaufwand der IT. Änderungen oder Erweiterungen am System sollen schnell und kostengünstig erfolgen. Gleichzeitig soll jedoch die Komplexität des Systems so wenig wie möglich erhöht werden [Hage03, S. 67].

Die Suffizienzstrategie fordert eine differenzierte Auseinandersetzung und ggf. eine Genügsamkeit gegenüber (Management-)Moden. Derartige Moden wie beispielsweise Total Quality Management, Lean Production, Virtuelle Unternehmen, Business Process Reengineering (BPR) usw. müssen kritisch hinterfragt werden [KiWa03, S. 410ff]. Es ist zu prüfen, ob sie im jeweiligen Unternehmen ihre Wirkung erzielen und zum Erfolg beitragen. Durch die Wiederverwendung bestehen- 
der Anwendungssoftware entsteht ein reduzierter Aufwand (Senkung der Kosten und Verkürzung der Entwicklungszeit) und erhöhte Qualität der technischen Komponenten [Stue02, S. 31ff].

Um die notwendigen Veränderungen innerhalb der Organisation und der IT erfolgreich zu bewältigen, sind Akzeptanz und Mitwirken aller Betroffenen an der Transformation notwendig (Partizipationsstrategie).

\subsection{Strategie der nachhaltigen Veränderung}

Nachhaltigkeitsstrategien haben nicht das Ziel, einen eindeutig zu spezifizierenden stabilen Endzustand zu erreichen. Vielmehr stellen sie ein Wertegefüge für einen gerichteten Prozess des Wandels dar [Cas ${ }^{+}$97]. Wichtig ist es jedoch, den Wandel so zu gestalten, dass er zu nachhaltigen Ergebnissen führt.

In der Literatur existieren verschiedene Phasenmodelle, die idealtypisch den Prozess des Wandels beschreiben [Rhei02, S. 303 ff.; Krue00, S. 273ff]. Aus der kritischen Auseinandersetzung mit diesen Modellen entwickelte SCHÄFFNER vier Kernbedingungen, deren Einhaltung nachhaltige Veränderungsprozesse kennzeichnen. Demnach müssen Veränderungen intendiert und indiziert sein, durch eine Weiterpflege unterstützt werden und ihre Ziele erreichen [Scha02, S. 306ff]. Diese Kriterien lassen sich sowohl auf organisatorische Veränderungen als auch auf Änderungen der IT-Architektur anwenden.

\subsection{Strategie der strukturierten Systemintegration}

Neue technische Entwicklungen wie das Internet und die damit verbunden Geschäftsmodelle innerhalb des E-Business stellen neue Anforderungen an die Funktionen der Unternehmens-IT. Die vollständige Neugestaltung der IT-Landschaft im Unternehmen wäre jedoch aus Wirtschaftlichkeitsgründen und den damit verbundenen Risiken oft nicht vertretbar. Bestehende IT-Systeme vieler Unternehmen (oftmals Legacy-Systeme) weisen im Kern die benötigten Funktionalitäten auf, die lediglich ergänzt oder anders angeordnet werden müssen. Die vorhandenen IT-Systemlandschaften sind jedoch durch eine hohe Anzahl von Abhängigkeiten zwischen Applikationen gekennzeichnet (Point to Point), die erheblich zur Komplexität des Gesamtsystems beitragen. Jede Änderung oder Erweiterung einer Applikation zieht eine große Zahl weiterer Änderungen anderer abhängiger Applikationen nach sich. Modifikationen werden somit teuer und schließlich unmöglich [Hage03, S. 69].

Aus diesem Grund empfiehlt sich eine Strategie, die nach einer systematischen Weiterverwendung bestehender Anwendungssysteme strebt (Suffizienzstrategie), zugleich jedoch die Komplexität der Systemlandschaft so weit reduziert, dass zukünftige Änderungen flexibel, effektiv und effizient durchführbar sind (Effizienz- 
strategie). Ein in diesem Zusammenhang viel diskutiertes Thema ist Enterprise Application Integration (EAI). Es stellt eine zentrale Plattform zur Verfügung, welche Anwendungen über entsprechende, zum Teil vorgefertigte Adapter anbindet (Konsistenzstrategie). Gegenüber der Point to Point Integration lässt sich somit eine erheblich kleinere Anzahl an Schnittstellen verwirklichen. Die strukturelle Komplexität wird dadurch deutlich verringert und der Grundstein für eine verbesserte Anpassungsfähigkeit gelegt.

Kern dieser Strategie ist also die Entwicklung von Integrations(Infra-)strukturen, welche es ermöglichen, Systeme mit anderen zu integrieren, ohne dabei jedes Mal die Gesamt-IT-Architektur in Frage zu stellen.

\subsection{Strategie der strukturellen Analogie von Organisations- und IT-Architektur}

Der Zusammenhang zwischen der Organisation einerseits und der IT andererseits hat in der wissenschaftlichen Literatur große Beachtung erfahren. Trotz der langjährigen Beschäftigung der Wissenschaft mit diesem Thema und den zahlreichen Veröffentlichungen lassen sich jedoch keine absoluten, verallgemeinernden Aussagen zu den Beziehungen zwischen IT und Organisation finden [MaRo88; LeHu98, S. 271f]. Das Bestehen monokausaler Zusammenhänge zwischen Organisation und IT wird heute weitgehend verneint. Vielmehr wird angenommen, dass Informationstechnologie eine den Gestaltungsspielraum des Organisators erweiternde Option darstellt - „IT als Enabler“ [Fres00, S. 142f].

Um eine Nachhaltigkeit der Unternehmensarchitektur zu erreichen, ist die Berücksichtigung dieser Abhängigkeiten in einer integrierten Gestaltung unabdingbar. Die Strategie der strukturellen Analogie soll die geforderte Stimmigkeit zwischen IT- und Organisationsarchitektur (Konsistenzstrategie) durch ihre strukturähnliche Gestaltung unterstützen [Gron03, S. 216; Wall96, S. 63].

IT- und Organisationsarchitekturen sind strukturanalog zueinander, wenn sie in ihren Merkmalsausprägungen weitgehend übereinstimmen. Besonders geeignet für den Strukturvergleich zwischen Organisations- und IT-Struktur erscheinen die Organisationsdimensionen Spezialisierung (Arbeitsteilung), Entscheidungsdelegation (Kompetenzverteilung) und Formalisierung. Wenig spezialisierte organisatorische Stellen oder Einheiten sollten durch entsprechend umfassende IT-Systeme unterstützt werden. Ebenso bedürfen wenig formalisierte Abläufe und Strukturen eher der Flexibilität von Groupware-Systemen als sehr stark formalisierte Abläufe, die besser durch Workflow-Systeme unterstützt werden.

Eine besondere Stellung nimmt die Dimension der Entscheidungsdelegation ein, welche in der Literatur vielfach unter den Schlagworten Zentralisierung und Dezentralisierung diskutiert wurde [Minz79, S. 189]. Primär betrachtet diese Dimension die Verteilung von Entscheidungsbefugnissen und -kompetenzen. Die Ent- 
scheidungsdelegation ist um so größer, je mehr Entscheidungsbefugnisse auf die unteren Hierarchieebenen verteilt werden [Beue92, Sp. 2616]. Dementsprechend sollten dezentrale Kompetenzen bzgl. fachlicher Entscheidungen auch mit dezentralen Kompetenzen bzgl. den dazu notwendigen IT-Systemen ausgestattet werden. Wie bei jeder dezentralen fachlichen Entscheidung auch, sollten sich dezentrale IT-Systeme an zentralen Vorgaben orientieren. Zentrale Vorgaben, Pläne oder Programme sind notwendig, um das Gesamtsystem zu koordinieren und um „Wildwuchs“ zu vermeiden.

Der Nachhaltigkeitsaspekt der strukturellen Analogie folgt mittelbar aus der Konsistenzstrategie, nach welcher IT- und Organisationsarchitektur in Übereinstimmung zueinander zu gestalten sind.

Entsprechend den zu bewältigenden fachlichen Aufgaben sind angemessene Organisationsstrukturen zu entwickeln. Beispielsweise kann es für ein global agierendes Unternehmen notwendig sein, Vertriebsaktivitäten dezentral aufzubauen und im Sinne sich selbst steuernder Teams wenig formalisierte Strukturen vorzugeben. Diese dezentralen Einheiten können dann sicherlich nicht sehr effizient mit IT-Systemen arbeiten, die zentral entwickelt wurden, lokale Besonderheiten nicht abbilden können und starre Prozesse vorgeben. Eine solche Unternehmensarchitektur folgt nicht der Konsistenzstrategie und wird auch nicht zur Nachhaltigkeit innerhalb der Unternehmung beitragen.

Im Kontext zunehmend zu beobachtender Zentralisierungsprozesse der IT stellt sich die Frage, wie dies den oft geforderten dezentralen Organisationsstrukturen im Sinne der strukturellen Analogie - entspricht. Es kann nicht zwingend geschlussfolgert werden, dass sich hier widersprüchliche Strukturen entwickeln. Dies kann mit der geschichtlichen Entwicklung der Unternehmungs-IT verdeutlicht werden, die mit dem vermehrten IT-Einsatz vor allem dezentral geprägt war. Selbst technisch eher als zentral zu beschreibende Großrechnersysteme wurden vor allem für isolierte dezentrale Einzelaufgaben wie die Lohn- und Gehaltsabrechnung eingesetzt, ohne Verknüpfungen mit anderen Unternehmungsfunktionen berücksichtigen zu müssen [Fres00, S. 129ff]. Im Gegensatz zur Koordination betriebswirtschaftlich-fachlicher Fragestellungen war eine unternehmensweite ITKoordination weder notwendig noch vorhanden. Erst mit der zunehmenden technischen und vor allem logischen Vernetzung von Systemen entlang von Geschäftsprozessen wurde auch eine zentrale Instanz zur Vorgabe eines unternehmensweiten Entscheidungsrahmens notwendig. Darum kann im Falle der IT weniger von einer Strukturverschiebung hin zu zentralen Strukturen gesprochen werden, sondern vielmehr von der Ausfüllung der bis dahin nicht vorhanden Funktion der unternehmensweiten IT-Koordination.

Eine Möglichkeit zur Realisierung der Strategie der strukturellen Analogie ist die Modularisierung der Unternehmensarchitektur. 


\subsection{Modularisierung - Strategie der Flexibilität und Referenz}

Ein aktuell diskutiertes Mittel zur Flexibilisierung und Komplexitätsreduktion innerhalb von IT- und Organisationsarchitekturen ist die Modularisierung innerhalb der Architekturen.

Flexibilität ist eine Voraussetzung für nachhaltige Architekturen, da sich nur flexible Architekturen dauerhaft an die Anforderungen ihrer Umwelt anpassen können. Die Forderung der Komplexitätsreduktion entstammt der entscheidungstheoretischen Sicht der Nachhaltigkeit. Danach stellen sich negativ irreversible Entscheidungen vor allem als Problem komplexer Systeme dar, in welchen nicht alle Wirkungen von Entscheidungen zur irreversiblen Ressourcenverwendung erkannt werden können [Krca03, S. 23f].

Nachfolgend werden die Grundgedanken der Modularisierung dargestellt. Dabei wird gezeigt, warum modulare Architekturen prinzipiell nachhaltig sein können. In Anlehnung an die im vorhergehenden Abschnitt beschriebene strukturelle Analogie zwischen Organisation und IT ist der Gedanke der Modularisierung auf beide Domänen anzuwenden.

\section{Modularisierung}

Ein Modul besteht aus zwei Teilen, der Modulschnittstelle und dem Modulrumpf. Die Modulschnittstelle enthält dabei die Spezifikation der Leistungen des Moduls und alle Annahmen, die für seine Umwelt zur „Benutzung“ notwendig sind. Der Modulrumpf implementiert die in der Schnittstelle spezifizierten Leistungen des Moduls.

Modularisierung bedeutet allgemein die Strukturierung eines Systems in relativ kleine, teilautonome und überschaubare Subsysteme (Module, Fraktale).

Die Komplexitätsreduktion ergibt sich dabei also aus der Subsystembildung innerhalb des Systems Unternehmung. Die Subsystembildung wirkt deswegen komplexitätsreduzierend, da sie zum einen die subsysteminterne Komplexität im Sinne der Kapselung vor der Subsystemumwelt verbirgt, zum anderen durch die Reduktion auf wenige bekannte Schnittstellen eine Entkopplung der Subsysteme im Sinne einer Reduktion von Abhängigkeiten bewirkt. Mit anderen Worten: Komplexität ist systemtheoretisch ein Selektionszwang und Systemdifferenzierung durch Subsystembildung eine Möglichkeit der Selektion zur Verringerung der Komplexität [Stue96, S. 73]. Im Zusammenhang damit steht die Fähigkeit der einzelnen Subsysteme, selbstständig zu agieren und sich selbst organisieren zu können ( $A u$ topoiesis). Dies entspricht der Forderung nach Dezentralisierung und Selbststeuerung.

Die Flexibilisierung der Strukturen und damit der Abläufe ergibt sich durch die nun leichtere Rekonfigurationsmöglichkeit der entkoppelten Module über ihre de- 
finierten Schnittstellen. Komplexitätsreduktion kann somit auch als Voraussetzung für die Flexibilisierung angesehen werden.

\section{Umsetzung}

Um eine effiziente Komplexitätsreduktion und eine angemessene Kombination zentraler und dezentraler Strukturen zu erreichen, schlagen AIER/SCHÖNHERR eine Modularisierung auf zwei Ebenen vor - dabei wird von der Ebene der Gesamtunternehmung als Makroebene und von der Ebene der Geschäftsprozesse als Mikroebene gesprochen (Abbildung 2).

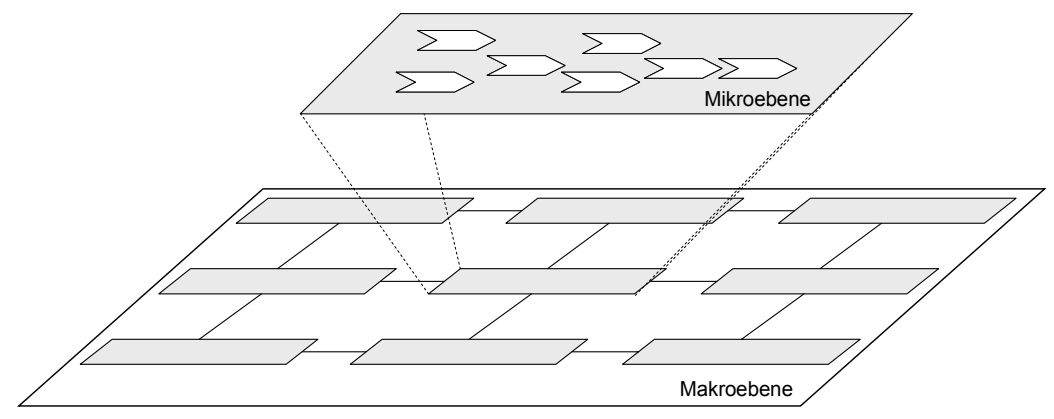

Abbildung 2: Ebenen der Modularisierung [AiSc03, S. 28]

Mit der Definition einer Makro- und einer Mikroebene werden zwei Ziele verfolgt:

1. Festigung und zugleich Flexibilisierung der Strukturen

2. Definition überschaubarer und managebarer Module

\section{Unterstützung der Nachhaltigkeit}

Die Makroebene hat eine stark strukturierende Wirkung. Die Module der Makroebene, die so genannten Makromodule, sollen über einen längeren Zeitraum stabil sein. Die Makroebene bildet damit ein Bezugssystem - eine Referenz - als Grundlage für die Gestaltung der Module der Mikroebene innerhalb der jeweiligen Makromodule. Die Module der Mikroebene hingegen sollen die Dynamik der wechselnden Prozessanforderungen durch entsprechende Rekonfiguration widerspiegeln - Flexibilität. Ziel ist es also, eine bezuggebende, stabile, organisationsinvariante Ebene - die Makroebene - und eine dynamische, flexibel konfigurierbare Ebene - die Mikroebene - zu bilden (Strategie der Flexibilität und Referenz).

Zwei grundlegende Prinzipien der Bildung von Modulen sind zum einen die Minimierung der externen Abhängigkeiten zwischen Modulen und zum anderen die Maximierung der internen Abhängigkeiten innerhalb der Module im Sinne einer Verschiebung von Abhängigkeiten in Module. 
Die Nachhaltigkeit wird also vor allem durch die organisationsinvariante stabile Makroebene unterstützt. Organisations- und IT-Anpassungen auf der Mikroebene haben keine Auswirkungen auf die Makroebene. Solange Schnittstellen unverändert bleiben, können durch die Entkopplung einzelne Module verändert oder ausgetauscht werden, ohne Änderungen an den übrigen Modulen vornehmen zu müssen. Durch die Verschiebung der Schnittstellen in die Module wird die Wahrscheinlichkeit, dass Schnittstellen zwischen Modulen auf der jeweiligen Betrachtungsebene angepasst werden müssen, reduziert. Ein Beispiel für ein Makromodul könnte „Vertrieb Privat- und Firmenkunden“ in einer Bank sein, ein dazugehöriges Mikromodul die „Kontoeröffnung““.

\section{Integration modularer IT}

Die vorgenannten Ansätze zur Modularisierung sind analog auf die Organisation und die IT anzuwenden (strukturelle Analogie). Für die technische Umsetzung kann beispielsweise eine angepasste zweistufige EAI-Architektur eingesetzt werden (Abbildung 3, vgl. zur Kombination weiterer Integrationstechnologien [AiSc04, S. 75f]). Innerhalb der Makromodule werden die einzelnen IT-Systeme bzw. Mikromodule über eine Integrationsplattform strukturiert angebunden. Zwischen den Makromodulen wird eine weitere Integrationsplattform eingesetzt. Dies ist möglich, da die Mehrzahl der Schnittstellen in die Module verschoben wurde.

Durch diese zweistufige Architektur werden Teile der Integrationsinfrastruktur dezentral gehalten, wodurch die lokalen Integrationsbedürfnisse besser berücksichtigt werden können als bei einer zentralen Plattform (Strategie der Partizipation), da bei Änderungsanforderungen weniger (vor allem die lokalen) Abhängigkeiten berücksichtigt werden müssen, solange es keine Änderungen in der zentralen EAI-Plattform auf der Makroebene gibt (Strategie der Flexibilität und Referenz).

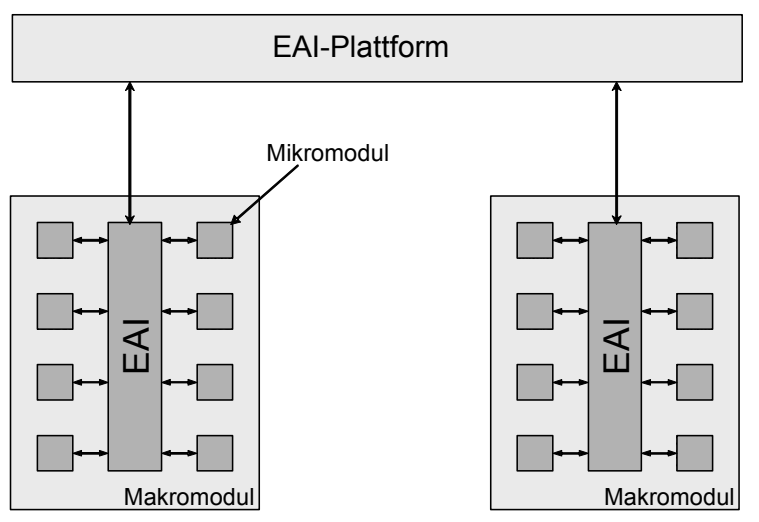

Abbildung 3: Zwei-Ebenen-EAI-Architektur 
Eine einzige zentrale Plattform ist in komplexen Architekturen meist nicht realisierbar. Zum einen ist das Integrationsszenario technisch und organisatorisch zu komplex, zum anderen scheitern solche Projekte oft an mikropolitischen Prozessen der Organisation.

\section{Indikatoren intern orientierter Nachhaltigkeit}

In diesem Abschnitt werden zuerst die Probleme der Umsetzung und der Zielerreichungskontrolle von Nachhaltigkeitsstrategien sowie die daraus resultierende Notwendigkeit von operativen Messgrößen besprochen. Anschließend wird ein Indikatorensystem zur Bewertung der Implementierung intern orientierter Nachhaltigkeitsstrategien vorgestellt.

\subsection{Notwendigkeit von Indikatoren}

Nachhaltigkeit ist ein schwer zu operationalisierendes Ziel. Der wichtigste Zweck nachhaltiger Systemgestaltung ist der Erhalt des Systems - hier der Unternehmung mit ihren wesentlichen Bestandteilen. Somit kann Nachhaltigkeit nur durch das Ende eines Systems falsifiziert werden. Darum wird meist von langfristiger Erhaltung des Systems gesprochen. Trotzdem ist die Beurteilung von Nachhaltigkeit weiterhin nur nachträglich möglich, da zukünftige Entwicklungen oft nur schwer prognostizierbar sind [Krca03, S. 18f].

Eine solche ex-post Bewertung ist in der Praxis nicht befriedigend. Um Nachhaltigkeitsstrategien erfolgreich zu implementieren, entsteht die Notwendigkeit, Indikatoren zu entwickeln, mit denen der Grad der Nachhaltigkeit zumindest abgeschätzt werden kann. Diese Indikatoren (sustainable performance indicators) sind messbare Kenngrößen, mit denen Ist- und Sollzustände beschrieben werden können. Sie bilden die Basis für ein Nachhaltigkeits-Management im Sinne eines Regelkreises. Die Indikatoren zeigen auf, wie erfolgreich die Planung und Implementierung der Unternehmensarchitektur im Hinblick auf die intern orientierte Nachhaltigkeit ist. Sie übernehmen die Rolle von Kennzahlen, mit denen ausgewählte betriebliche Nachhaltigkeitsleistungen bzw. Nachhaltigkeitsdimensionen (IT- und Organisationsarchitektur und ihre Wechselwirkung) überprüft werden können.

\subsection{Bestimmung eines Indikatorensystems}

Um die Nachhaltigkeit der Unternehmensarchitektur zu bewerten, muss zunächst eine adäquate Auswahl von Indikatoren bestimmt werden. Die Gesamtheit von operativ überprüfbaren Indikatoren zur Beurteilung nachhaltiger Entwicklung wird als Indikatorensystem bezeichnet. Nachfolgend werden die wesentlichen In- 
dikatoren aufgeführt, die nach Ansicht der Autoren die Basis für ein adäquates Managementinstrument bilden, um die Implementierung der vorgestellten Nachhaltigkeitsstrategien beurteilen und beeinflussen zu können. Dazu werden zuerst sechs Indikatorencluster vorgestellt und anschließend für ein Cluster konkrete Messgrößen vorgeschlagen. Für die praktische Anwendung ist es notwendig, diese Indikatoren situativ zu ergänzen.

\section{Standardisierungsgrad}

Dieses Indikatorencluster bezieht sich auf den Grad der Nutzung von Standards in Organisations- und IT-Architekturen. Auf Seiten der Organisationsarchitektur ist hier vor allem die Verwendung von Prozessstandards und Referenzmodellen zu nennen. Bezogen auf die IT-Architektur ist die Festlegung und Kontrolle von Daten- und Technologiestandards, z.B. in Technology Sets, messbar. Die Integration von Prozess- und Datenstandards erfolgt z.B. in Standards, wie sie mit der Unified Modeling Methodology (UMM) in ebXML spezifiziert werden können [Diet03, S. $117 \mathrm{ff}]$.

\section{Generizitätsgrad}

Dieses Indikatorencluster bezieht sich auf die Fähigkeit von Systemelementen durch Vorgaben von ,neutralen Lösungen“ ein hohes Maß an Kompatibilität aufzubauen. Die Systemelemente sind besser miteinander vereinbar und die Integrationsfähigkeit von einzubindenden Elementen wird gesteigert. In der Domäne der IT-Architektur bezieht sich dieser Indikator auf die Interoperabilität und Austauschbarkeit von einzelnen IT-Elementen. Die Unterstützung von TCP/IP oder XML erhöhte beispielsweise die Systembindung durch die Bereitstellung von neutralen, allgemein gültigen Lösungen - die Integrationsfähigkeit nimmt zu.

\section{Transparenzgrad}

Der Transparenzgrad indiziert das unternehmensweite Wissensrepertoire, so z.B. schriftlich fixierte Prozesse, Regeln, Richtlinien, Verantwortlichkeiten, Kommunikationskanäle, Modelle usw. Der Transparenzgrad ist sowohl für die Organisations- als auch die IT-Domäne von Bedeutung. Ein hoher Transparenzgrad gibt sowohl qualitativen als auch quantitativen Aufschluss über die Unternehmensarchitektur. Dies ist eine Voraussetzung für nachhaltige Entscheidungen.

\section{Modularitätsgrad}

Der Modularitätsgrad beschreibt, wie ausgeprägt die Modulbildung ist, wie stark die Entkopplung, die Autonomie, die Selbstorganisation, die Wiederverwendbarkeit und die Austauschbarkeit von Modulen und letztlich die Konsolidierung der Architekturen ist. Die genannten Punkte gelten dabei analog für die Organisationsund die IT-Architektur. Die Übereinstimmung der beiden Teilarchitekturen bezeichnet den Grad der strukturellen Analogie dieser Architekturen. 


\section{Organisatorische Verankerung}

Die nachhaltige Unternehmensentwicklung bedarf der Implementierung von organisatorischen Stellen, die hier als Unternehmensarchitekten bezeichnet werden. Ihre Aufgaben umfassen die Planung, Implementierung und Kontrolle der Unternehmensarchitektur u.a. in Bezug auf ihre Nachhaltigkeit. Den Unternehmensarchitekten kommt die Rolle eines Machtpromotors zu. Sie sind diejenigen, die dem Transformationsprozess der Unternehmensarchitektur im Hinblick auf die Erreichung der obersten Nachhaltigkeitsziele - hier als Nachhaltigkeitsstrategien bezeichnet, Ressourcen verschaffen. Notwendigerweise muss der Unternehmensarchitekt selbst mit entsprechender Macht ausgestattet sein.

\section{Experteneinschätzungen}

Dieses „weiche“ Indikatorcluster spiegelt qualitative Expertisen der organisatorischen und informationstechnischen „Ist-Situationen“ wider. Die befragten Personen sind vornehmlich die jeweiligen unternehmensinternen Experten, die täglich mit der relevanten Domäne im Detail befasst sind. Ergänzend können auch externe Experten befragt und ihr Wissen herangezogen werden. Ihre Einschätzungen umfassen beispielsweise das Potenzial, neue IT-Systeme zu integrieren, das Potenzial neue Produkte und Prozesse abzubilden, oder generelle Beurteilungen über die Wandlungsfähigkeit der IT- und Organisationsarchitektur. Die Korrelation von unterschiedlichen Experteneinschätzungen kann als ein Maß für die qualitative Beurteilung der Unternehmensarchitektur herangezogen werden.

Für das Indikatorencluster Modularitätsgrad können beispielsweise folgende Indikatoren herangezogen werden:

- Verhältnis der modulinternen Schnittstellen zur gesamten Anzahl der Schnittstellen (jeweils für Organisation/IT)

- Anzahl der Prozessvarianten innerhalb von Modulen

- Überschneidungsgrad von Prozessen in unterschiedlichen Modulen

- Projektaufwand (z.B. in Personentagen) zur Implementierung neuer Prozesse

- Anteil der plattformunabhängigen Systeme

Die genannten Indikatoren sind weiter situativ zu präzisieren. So kann der Indikator der Plattformunabhängigkeit genauer beschrieben werden in:

- Unabhängigkeit von verwendeter Hardware

- Unabhängigkeit von verwendeten Betriebssystemen

- Unabhängigkeit von verwendeten Datenbanksystemen

- Unabhängigkeit von verwendeten Applikationsservern

- Unabhängigkeit von verwendeten Services wie Berechtigungsservices etc. 


\subsection{Beurteilung der Strategien}

Nachdem die ausgewählten Indikatoren gemessen wurden, sollten diese aggregiert werden und zu den implementierten Strategien in Relation gestellt werden, um den Erfolg einer jeden Strategie beurteilen zu können und Handlungsbedarfe zu identifizieren. Dies kann z.B., wie in Tabelle 1 generisch dargestellt, erfolgen, indem zuerst die Ausprägungen der Indikatoren erhoben werden, diese normiert und über ein Gewicht den einzelnen Strategien zugeordnet werden. Daraus ergibt sich eine Bewertung der Implementierung der Nachhaltigkeitsstrategien (Abbildung 4).

\begin{tabular}{|c|c|c|c|c|c|c|c|c|c|c|c|c|c|}
\hline \multicolumn{4}{|c|}{ Indikatoren } & \multicolumn{10}{|c|}{ Strategien } \\
\hline \multirow[b]{3}{*}{$\mathrm{Nr}$} & \multirow[b]{3}{*}{ Bezeichnung } & \multirow[b]{3}{*}{ Ausprägung } & \multirow{3}{*}{$\begin{array}{l}\text { Wert } \\
\text { normiert } \\
(0-9)\end{array}$} & \multirow{2}{*}{\multicolumn{2}{|c|}{$\begin{array}{l}\mathrm{S}_{1} \\
\text { Strategie } 1 \\
\end{array}$}} & \multicolumn{2}{|l|}{$\mathrm{S}_{2}$} & \multicolumn{2}{|l|}{$S_{3}$} & \multicolumn{2}{|l|}{$S_{4}$} & \multicolumn{2}{|l|}{$S_{5}$} \\
\hline & & & & & & Strategie & & Strategie & & Strategie & & Strategie & \\
\hline & & & & \begin{tabular}{|l} 
Gewicht \\
$(\%)$
\end{tabular} & \begin{tabular}{|l|}
$\begin{array}{l}\text { Wert } \\
\text { gewichtet }\end{array}$ \\
\end{tabular} & \begin{tabular}{|l}
$\begin{array}{l}\text { Gewicht } \\
(\%)\end{array}$ \\
\end{tabular} & \begin{tabular}{|l|}
$\begin{array}{l}\text { Wert } \\
\text { gewichtet }\end{array}$ \\
\end{tabular} & \begin{tabular}{|l}
$\begin{array}{l}\text { Gewicht } \\
(\%)\end{array}$ \\
\end{tabular} & \begin{tabular}{|l|}
$\begin{array}{l}\text { Wert } \\
\text { gewichtet }\end{array}$ \\
\end{tabular} & \begin{tabular}{|l|}
$\begin{array}{l}\text { Gewicht } \\
(\%)\end{array}$ \\
\end{tabular} & $\begin{array}{l}\text { Wert } \\
\text { gewichtet }\end{array}$ & \begin{tabular}{|l}
$\begin{array}{l}\text { Gewicht } \\
(\%)\end{array}$ \\
\end{tabular} & \begin{tabular}{|l} 
Wert \\
gewichtet
\end{tabular} \\
\hline$T_{1}$ & Indikator 1 & 100 & & $10 \%$ & 0,3 & $0 \%$ & $\overline{0}$ & $20 \%$ & $\overline{0,6}$ & $10 \%$ & $\overline{0,3}$ & $5 \%$ & 0,15 \\
\hline$I_{2}$ & Indikator 2 & 12 & & $0 \%$ & 0 & $5 \%$ & 0,3 & $30 \%$ & 1,8 & $10 \%$ & 0,6 & $5 \%$ & 0,3 \\
\hline$I_{3}$ & Indikator 3 & hoch & & $50 \%$ & 3,5 & $30 \%$ & 2,1 & $0 \%$ & 0 & $50 \%$ & 3,5 & $40 \%$ & 2,8 \\
\hline$I_{4}$ & \begin{tabular}{|l|} 
Indikator 4 \\
\end{tabular} & sehr hoch & & $20 \%$ & 1,8 & $25 \%$ & 2,25 & $20 \%$ & 1,8 & $20 \%$ & 1,8 & $20 \%$ & 1,8 \\
\hline$I_{5}$ & Indikator 5 & 4 & & $10 \%$ & 0,8 & $10 \%$ & 0,8 & $10 \%$ & 0,8 & $10 \%$ & 0,8 & $10 \%$ & 0,8 \\
\hline$I_{6}$ & Indikator 6 & 25 & & $0 \%$ & 0 & $20 \%$ & 1,4 & $10 \%$ & 0,7 & $0 \%$ & 0 & $10 \%$ & 0,7 \\
\hline 17 & Indikator 7 & 10 & & $10 \%$ & 0,5 & $10 \%$ & 0,5 & $10 \%$ & 0,5 & $0 \%$ & 0 & $10 \%$ & 0,5 \\
\hline & Strat & egieimpleme & ntierung & & 6,9 & & 7,35 & & 6,2 & & 7 & & 7,05 \\
\hline
\end{tabular}

Tabelle 1: Bewertungsmatrix zur Nachhaltigkeit

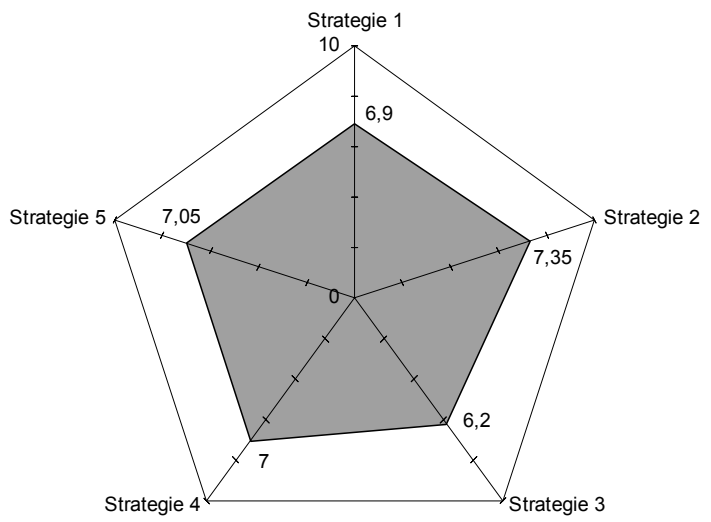

Abbildung 4: Erfolgsbewertung der Nachhaltigkeitsstrategien

Bei der konkreten Indikatorenauswahl ist besonders auf die Abhängigkeiten zwischen Indikatoren zu achten. Prinzipiell wird es kaum möglich sein, vollständig überschneidungsfreie bzw. voneinander unabhängige Indikatoren zu spezifizieren. Dennoch sollte versucht werden, diese Abhängigkeiten zu minimieren bzw. ein Bewusstsein dafür zu schaffen. Beispielweise weisen die Indikatoren „Anteil dokumentierter Schnittstellen“ und „Wiederverwendungsfähigkeit von Softwarekomponenten" eine große Überschneidung auf, was zu verzerrten Ergebnissen bei 
der Strategiebewertung führt. Es wird jedoch nicht als sinnvoll erachtet, diese Abhängigkeit im Modell zu berücksichtigen, da hierdurch die Modellkomplexität steigt und die Anwendbarkeit sinkt. Letztlich sind die sich aus dem Modell ergebenden Zahlenwerte selbst wieder als Indikatoren zu verstehen. Sie geben also vor allem Tendenzen wieder und sollen Handlungsbedarfe offen legen. Ergebnisse eines zu komplexen und damit nicht nachvollziehbaren Modells werfen u.U. Akzeptanzprobleme auf, was der Partizipationsstrategie widerspricht.

\section{Zusammenfassung}

In der Ökologie und den Sozialwissenschaften ist die Diskussion um Nachhaltigkeit seit Jahren etabliert. Im Bereich der Betriebswirtschaftslehre oder Informatik gibt es nur wenige Veröffentlichungen, die Nachhaltigkeit nicht unter primär ökologischen Aspekten diskutieren. Die vielfältige Verwendung des Begriffs ist darüber hinaus in der Regel unreflektiert. Dieser Beitrag bezieht Nachhaltigkeit im Rahmen der Wirtschaftsinformatik auf ganzheitlich betrachtete Unternehmensarchitekturen und versucht somit, die beschriebene Situation zu ändern.

Die ökologisch geprägte Nachhaltigkeitsdiskussion gibt erste Hinweise auf einen allgemeinen, aber immer noch primär extern orientierten Nachhaltigkeitsbegriff. Bislang fehlt jedoch eine Definition eines intern orientierten Nachhaltigkeitsbegriffs. Kern einer möglichen Definition ist bislang lediglich der bewusste Umgang mit den Ressourcen der Unternehmung im Kontext des stetigen organisatorischen und technischen Wandels. Vor allem jedoch fehlt es an allgemein gültigen operativen Handlungsanweisungen zur Erreichung und Bewertung nachhaltiger Unternehmensarchitekturen.

In diesem Beitrag wurden darum zuerst Strategien zur Erreichung nachhaltiger Architekturen vorgeschlagen. Anschließend wurde ein Indikatorensystem umrissen, mit welchem der Erfolg der Strategieimplementierung bewertet werden kann. Dieses Indikatorensystem steht jedoch erst am Anfang und bedarf für den praktischen Einsatz der weiteren Konkretisierung.

\section{Literatur}

[AiSc03] Aier, S., Schönherr, M.: Flexibilisierung von Organisations- und IT-Architekturen durch EAI; in: Aier, S., Schönherr, M. (Hrsg): Enterprise Application Integration Management komplexer Architekturen, Berlin 2003: Gito. Reihe: Enterprise Architecture, Band 1, S. 3-56. 
[AiDo04] Aier, S., Dogan, T.: Nachhaltigkeit als Gestaltungsziel von Unternehmensarchitekturen; in: Aier, S., Schönherr, M. (Hrsg): Enterprise Application Integration - Serviceorientierung und nachhaltige Architekturen, Berlin 2004: Gito. Reihe: Enterprise Architecture, Band 2, noch nicht erschienen.

[AiSc04] Aier, S., Schönherr, M.: Enterprise Application Integration als Enabler flexibler Unternehmensarchitekturen; in: Hasselbring, W., Reichert, M. (Hrsg): EAI-Workshop 2004 - Enterprise Application Integration, Berlin 2004: Gito, S. 69-78.

[Bas+03] Bass, L., Clements, P., Kazman, R.: Software Architecture in Practice; 2. Auflage, Boston 2003: Pearson Education Inc.

[Beue92] Beuermann, G.: Zentralisation und Dezentralisation; in: Frese, E. (Hrsg): Handwörterbuch der Organisation. 3. Auflage, Stuttgart 1992: Poeschel, Sp. 2611-2625.

[Blei91] Bleicher, K.: Organisation: Strategien, Strukturen, Kulturen; 2. Auflage, Wiesbaden 1991: Gabler.

[Cas+97] Castle, E., Berrens, R., Polasky, S.: The Economics of Sustainability; Natural Resources Journal, 36. Jg. (1997), Nr. 4, S. 475-491.

[Conr00] Conrad, J.: Nachhaltige Entwicklung - einige begriffliche Präzisierungen oder der heroische Versuch einen Pudding an die Wand zu nageln; FFU-Report 2000. http://www.fu-berlin.de/ffu/download/rep_00-07.PDF, Zugriff am 10.02.2004.

[Diet03] Dietrich, J.: Bedeutung von B2BStandards für die Konzeption interner Integrationsszenarien im Hinblick auf eine verbesserte Integration in Wertschöpfungsnetzen; in: Aier, S., Schönherr, M. (Hrsg): Enterprise Application Integration - Management komplexer Architekturen, Berlin 2003: Gito. Reihe: Enterprise Architecture, Band 1, S. 117-146.

[Fres00] Frese, E.: Grundlagen der Organisation: Konzept - Prinzipien - Strukturen; 8. Auflage, Wiesbaden 2000: Gabler.

[FrSt02] Frese, E., Stöber, H. (Hrsg). E-Organisation. Wiesbaden 2002: Gabler.

[Gait83] Gaitanides, M.: Prozeßorganisation; München 1983: Vahlen.

[Gron03] Gronau, N.: Wandlungsfähige Informationssystemarchitekturen - Nachhaltigkeit bei organisatorischem Wandel; Berlin 2003: Gito.

[Hage03] Hagen, C.: Integrationsarchitektur der Credit Suisse; in: Aier, S., Schönherr, M. (Hrsg): Enterprise Application Integration - Management komplexer Architekturen, Berlin 2003: Gito. Reihe: Enterprise Architecture, Band 1, S. 61-83.

[HaHu01] Hahn, D., Hungenberg, H.: PuK - Wertorientierte Controllingkonzepte; 6. Auflage, Wiesbaden 2001: Gabler.

[Hube95] Huber, J.: Nachhaltige Entwicklung durch Suffizienz, Effizienz und Konsistenz; in: Fritz, P., Huber, J., Levi, H. W. (Hrsg): Nachhaltigkeit in naturwissenschaftlicher und sozialwissenschaftlicher Perspektive, Stuttgart 1995: Hirzel, S. 31-46.

[KiKu92] Kieser, A., Kubicek, H.: Organisation; 3. Auflage, Berlin, New York 1992: De Gruyter. 
[KiWa03] Kieser, A., Walgenbach, P.: Organisation; 4. Auflage, Stuttgart 2003: SchäfferPoeschel.

[Krca03] Krcal, H.-C.: Systemtheoretischer Metaansatz für den Umgang mit Komplexität und Nachhaltigkeit; in: Leisten, R., Krcal, H.-C. (Hrsg): Nachhaltige Unternehmensführung - Systemperspektiven, Wiesbanden 2003: Gabler, S. 3-30.

[Krcm90] Krcmar, H.: Bedeutung und Ziele von Informationssystem-Architekturen; Wirtschaftsinformatik, 32. Jg. (1990), Nr. 5, S. 395-402.

[Krue98] Krüger, W.: Management permanenten Wandels; in: Glaser, H., Schröder, E. F., Werder, A. v. (Hrsg): Organisation im Wandel der Märkte, Wiesbaden 1998: Gabler, S. 227-249.

[Krue00] Krüger, W.: Organisationsmanagement: Vom Wandel der Organisation zur Organisation des Wandels; in: Frese, E. (Hrsg): Organisationsmanagement - Neuorientierung der Organisationsarbeit, Stuttgart 2000: Schäffer-Poeschel, S. 271-304.

[LeSt03] Leitschuh-Fecht, H., Steger, U.: Wie wird Nachhaltigkeit für Unternehmen attraktiv? - Business Case für nachhaltige Unternehmensentwicklung; in: Linne, G., Schwarz, M. (Hrsg): Handbuch Nachhaltige Entwicklung, Opladen 2003: Leske + Budrich, S. 257-266.

[LeHu98] Lewin, A. Y., Hunter, S. D.: Information Technology \& Organizational Design: A Longitudinal Study of Information Technology Implementations in the U.S. Retailing Industrie, 1980-1996; in: Glaser, H., Schröder, E. F., Werder, A. v. (Hrsg): Organisation im Wandel der Märkte, Wiesbaden 1998: Gabler, S. 251-286.

[MaRo88] Markus, M., Robey, D.: Information Technology and Organizational Change: Causal Structure in Theory and Research; Management Science, 34. Jg. (1988), Nr. 5, S. 583-589.

[Minz79] Mintzberg, H.: The Structuring of Organizations: A Synthesis of the Research; Reihe: The theory of management policy, Englewood Cliffs, NJ 1979: Prentice-Hall.

[Morg97] Morgan, G.: Images of organization; 2nd. Auflage, Thousand Oaks, Calif. 1997: Sage Publications.

[Nad+94] Nadler, D. A., Gerstein, M. S., Shaw, R. B.: Organisationsarchitektur; Frankfurt/Main, New York 1994: Campus.

[Neu+00] Neumann, S., Probst, C., Wernsmann, C.: Kontinuierliches Prozessmanagement; in: Becker, J., Kugeler, M., Rosemann, M. (Hrsg): Prozessmanagement - Ein Leitfaden zur prozessorientierten Organisationsgestaltung, Berlin 2000: Springer, S. 263-282.

[Rhei02] Rhein, R.: Betriebliche Gruppenarbeit im Kontext der lernenden Organisation; Reihe: Bildung und Organisation, Band: 9, Frankfurt am Main et al. 2002: Lang.

[Scha02] Schäffner, L.: Der Beitrag der Veränderungsforschung zur Nachhaltigkeit von Organisationsentwicklung; München et al. 2002: Hampp.

[Stue96] Stünzer, L.: Systemtheorie und betriebswirtschaftliche Organisationsforschung; Berlin 1996: Duncker \& Humblot (zugl. Dissertation, Universität Trier, 1996). 
[Stue02] Stützle, R.: Wiederverwendung ohne Mythos: Empirisch fundierte Leitlinien für die Entwicklung wiederverwendbarer Software. Dissertation, Technische Universität München 2002.

[Teic02] Teichert, V.: Indikatoren zur Lokalen Agenda 21: ein Modellprojekt in sechzehn Kommunen; Reihe: Indikatoren und Nachhaltigkeit, Band: 1, Opladen 2002: Leske + Budrich.

[Wall96] Wall, F.: Organisation und betriebliche Informationssysteme - Elemente einer Konstruktionslehre; Wiesbaden 1996: Gabler (zugl. Habilitation, Universität Hamburg, 1996).

[Worl87] World Commission on Environment and Development: Our common future; Oxford, New York 1987: Oxford University Press. 